\title{
I-GEL AS AN AIRWAY DEVICE- A PROSPECTIVE STUDY IN 30 PLASTIC SURGICAL PATIENTS
}

\author{
Aniruddha Nirkhi' ${ }^{1}$ Ketaki Patwardhan², Vijay Patil ${ }^{3}$
}

${ }^{1}$ Consultant Cardiac Anaesthetist, Bombay Hospital, Mumbai.

${ }^{2}$ Associate Professor, Department of Anaesthesia, Rajiv Gandhi Medical College and Chhatrapati Shivaji Maharaj Hospital, Kalwa, Thane.

${ }^{3}$ Associate Professor, Department of Anaesthesia, Rajiv Gandhi Medical College and Chhatrapati Shivaji Maharaj Hospital, Kalwa, Thane.

\section{ABSTRACT}

\section{BACKGROUND}

Supraglottic airway devices have become invaluable for both routine and difficult airway management. After the introduction of the laryngeal mask airway LMA-Classic in the 1980s, there has been a steady increase in the applications for use of supraglottic airways as well as incidence of use. I-gel is an innovative supraglottic airway device. Made from a medical grade thermoplastic elastomer, Igel has been designed to create a non-inflatable, anatomical seal of the pharyngeal, laryngeal and perilaryngeal structures whilst avoiding compression trauma.

The aim of this study is to determine the ease of insertion, adequacy of ventilation upon insertion, the haemodynamic stability during and after insertion and postoperative complications occurring due to I-gel insertion.

\section{MATERIALS AND METHODS}

We conducted a prospective randomised study on I-gel TM (Intersurgical Ltd, Wokingham, UK) on 30 plastic surgical patients of ASA physical status I-II. After premedication with midazolam and fentanyl, induction was done with Propofol and I-gel was inserted according to manufacturer's instruction. ${ }^{1}$ An effective airway was confirmed by bilateral chest movement, square wave on capnograph and $\mathrm{SpO}_{2}>95 \%$.

\section{RESULTS}

The study shows that I-gel has many advantages, including ease of insertion, higher success rate on first attempt of insertion, effective airway maintenance, ease of gastric tube insertion, less number of failures and lesser complications intraoperatively as well as postoperatively.

\section{CONCLUSION}

I-gel is a simple, excellent and easy-to-use supraglottic airway device, easy to insert without the need of many manipulations with maintenance of airway.

\section{KEYWORDS}

Supraglottic, I-gel, Airway, Haemodynamic.

HOW TO CITE THIS ARTICLE: Nirkhi A, Patwardhan K, Patil V. I-gel as an airway device- A prospective study in 30 plastic surgical patients. J. Evolution Med. Dent. Sci. 2017;6(66):4779-4782, DOI: 10.14260/Jemds/2017/1035

\section{BACKGROUND}

Since 1983 there was no update on the cuffed supraglottic airway devices, which is introduced blindly into the hypopharynx to form a seal around the larynx, so permitting spontaneous or positive pressure ventilation without penetration of the larynx or oesophagus. It was used in place of the facemask in routine anaesthesia, and where difficulties with the airway were expected. ${ }^{1}$

Supraglottic airway devices are developed with increasing frequency following the overwhelming success of the laryngeal mask airway (LMA). The LMA and similar supraglottic airway devices use an inflatable cuff to wedge into the upper oesophagus and provide a perilaryngeal seal. ${ }^{2}$

Financial or Other, Competing Interest: None.

Submission 21-06-2017, Peer Review 06-08-2017,

Acceptance 12-08-2017, Published 17-08-2017.

Corresponding Author:

Dr. Ketaki Patwardhan,

Flat No. 1C, Angel Apartment,

Cosmos Springs 2,

Ovala, Ghodbunder Road, Thane.

E-mail: ketaki_p18@yahoo.co.in

DOI: $10.14260 /$ jemds $/ 2017 / 1035$
One of the most limiting features of the LMA is a lack of airway protection from regurgitated gastric contents, controversy surrounds the theory that the presence of the LMA in the pharynx could promote regurgitation by reducing lower oesophageal sphincter tone. ${ }^{3}$

I-gel TM (Intersurgical Ltd, Wokingham, U.K.) is a new supraglottic airway device with anatomically designed, noninflatable mask, which is soft, gel like and transparent, made of thermoplastic elastomer. The soft, non-inflatable cuff fits snugly onto the perilaryngeal framework and its tip lies in the proximal opening of the oesophagus, isolating the oropharyngeal opening from the laryngeal inlet. The outer cuff shape ensures that the blood flow to the laryngeal and perilaryngeal framework is maintained and helps the possibility to reduce neurovascular compression trauma to the nerves. The device has buccal cavity stabiliser which has propensity to adopt its shape to oropharyngeal curvature of the patients. It is anatomically widened and concaved to eliminate the potential for rotation, thereby reducing the risk of malposition. This buccal cavity stabiliser houses airway tubing and separate gastric channel. The tube section is firmer than the soft bowl of the gastric channel. The firmness of tube section and its natural oropharyngeal curvature allows the 
device to be inserted by grasping the proximal end of it against the hard palate into the pharynx without inserting the fingers into the mouth of the patients. The smooth contiguous surface of the device from the tip of the bowl to the proximal end of the tube, allows the device to easily slide posteriorly along the hard palate, pharynx and hypopharynx. The device has integral bite block which is marked with a horizontally placed black line, which acts as a guide to depth of insertion. The device also has a channel for gastric tube drain (Except size 1), which runs through the device from its proximal opening at the slide of flat connector wing to the distal tip of the noninflatable mask. The gastric channel allows suction, detection of leak and passage of gastric tube. The maximum size of gastric tube which can be passed through different sizes is given in Table 1. The device also has an epiglottic blocker which prevents down folding of the epiglottis and obstruction of the distal opening airway. 4

\begin{tabular}{|c|c|c|c|}
\hline $\begin{array}{c}\text { Size of } \\
\text { I-gel }\end{array}$ & $\begin{array}{c}\text { Body } \\
\text { Weight (kg) }\end{array}$ & $\begin{array}{c}\text { Maximum Size } \\
\text { of Gastric } \\
\text { Tube }\end{array}$ & $\begin{array}{c}\text { Maximum Size of } \\
\text { ETT which can } \\
\text { be Passed }\end{array}$ \\
\hline 1 & $1-5$ & N/A & 3.5 \\
\hline 1.5 & $5-12$ & 10 & 4.5 \\
\hline 2 & $10-25$ & 12 & 5.0 \\
\hline 2.5 & $25-35$ & 12 & 6.0 \\
\hline 3 & $30-60$ & 12 & 6.0 \\
\hline 4 & $50-90$ & 12 & 7.0 \\
\hline 5 & $90+$ & 14 & 8.0 \\
\hline \multicolumn{4}{|c|}{ Table 1. Recommended Size of I-gel, Maximum Size of } \\
Gastric Tube and Endotracheal tube \\
which can be Inserted through it.
\end{tabular}

\section{METHODS}

Thirty consecutive patients of either sex belonging to ASA physical status I- II were recruited for this study after approval from institutional ethical committee with informed written consent.

\section{Inclusion Criteria}

- Weight: 50 - $90 \mathrm{Kg}$.

- $\quad$ ASA: I -II.

- Elective plastic surgery in the supine position.

\section{Exclusion Criteria}

1. Any pathology of the neck, upper respiratory tract or upper alimentary tract.

2. Predicted difficult airway, mouth opening $<2.0 \mathrm{~cm}$, the view of the oropharynx on opening the mouth will be scored by the method of Mallampati as modified by Samsoon and Young.

3. A body mass index $>35 \mathrm{~kg} / \mathrm{m}^{2}$.

4. Patients with history of obstructive sleep apnoea.

5. Trendelenburg position.

6. History of lung diseases.

7. Potentially full stomach patients (trauma, morbid obesity, pregnancy, history of gastric regurgitation and heart burn).

8. Oesophageal reflux (hiatus hernia).

In the operation theatre, an IV line was secured and monitors were applied which included ECG, pulse oximetry and capnography. Induction of anaesthesia in each case was done in supine position with the head on the standard pillow.
Patients were given, Inj. Glycopyrrolate $0.04 \mathrm{mg} \mathrm{kg}^{-1}$, Inj. Ondansetron $0.08 \mathrm{mg} \mathrm{kg}^{-1}$, Inj. Fentanyl $2 \mu \mathrm{g} . \mathrm{kg}^{-1}$ and Inj.Midazolam0.02mg.kg-1. After preoxygenation for 3 minutes, each patient received induction dose of Inj. Propofol 2-2.5 mg kg-1 over 30-40 seconds with endpoint of induction being loss of eyelash reflex. Facemask ventilation was done with $40 \% \mathrm{O}_{2}$ in $\mathrm{N}_{2} \mathrm{O}$ and $1-1.5 \%$ of Sevoflurane until optimal conditions for I-gel insertion were attained (Jaw relaxation, no movement). Additional increments of Inj. Propofol were given as and when required until level of anaesthesia adequate for insertion of device was achieved. The insertion of I-gel was done according to manufacturer's instruction. The front, back and sides of the cuff were lubricated with water based jelly. The patient's head was placed in 'sniffing the morning air' position. The lubricated device was grasped along the integral bite block and was introduced into the mouth in the direction towards the hard palate and was glided downwards and backwards along the hard palate until definite resistance was felt. The device was connected to breathing circuit and patient ventilated manually. An effective airway was confirmed by bilateral symmetrical chest movement, square waveform on capnograph and normal $\mathrm{SpO}_{2}$ (>95\%). The device was secured with adhesive tape. If the airway was not effective, manipulations were done in the form of increasing the depth of insertion, giving jaw thrust or chin lift or changing size of the device. If it was not possible to maintain an effective airway after 3 insertion attempts; the device was taken as failure and alternative technique (ProSeal- LMA or ETT) was used. Ventilation of patient was manually assisted until the spontaneous breathing resumed. Anaesthesia was maintained on $\mathrm{O}_{2}: \mathrm{N}_{2} \mathrm{O}(40: 60 \%)$ and Isoflurane/Sevoflurane (1-1.5\%) with spontaneous ventilation.

\section{Intraoperative- In all Patients, Monitoring was divided in Two Groups- \\ Group I- Standard monitoring- \\ - $\quad$ ECG. \\ - Pulse oximetry. \\ - Arterial blood pressure monitoring. \\ - $\quad \mathrm{ETCO}_{2}(\mathrm{mmHg})$}

\section{Group II}

1. Ease of insertion was noted. An easy insertion was defined as insertion within the pharynx without resistance in a single manoeuvre. A difficult insertion was one in which there was resistance to insertion or where more than one manoeuvre was required to seat the device within the pharynx.

2. Insertion time (The time between picking up the device and obtaining an effective airway).

3. Attempts (1, 2, 3, failure). Three insertion attempts were allowed before a failure of insertion was recorded. If the I-gel could not achieve a satisfactory airway within three insertions either a LMA-Classic was inserted or the trachea was intubated conventionally. A satisfactory placement was noted if the expired tidal volume was more than $8 \mathrm{ml} / \mathrm{kg}$ and there was no drainage tube leak.

4. Ease of gastric tube insertion (Number of attempts).

5. Any regurgitation of fluid through the gastric channel or airway tube.

6. Assessment of chest compliance.

7. Any airway obstruction, partial or complete. 
Postoperative- After Smooth Recovery of the Patients the Data Recorded was-

- Patient removes the device by himself (awake removal).

- Blood on device.

- Dysphagia/ Dysphonia.

- Nausea and vomiting.

- Trauma of the mouth, tooth or pharynx.

\section{RESULTS}

1. Age

\begin{tabular}{|c|c|c|}
\hline Age Group (years) & No. of Patients & Percentage (\%) \\
\hline$<20$ & 2 & 6.67 \\
\hline $20-40$ & 17 & 56.66 \\
\hline $40-60$ & 7 & 23.33 \\
\hline$>60$ & 4 & 13.34 \\
\hline Total & $\mathbf{3 0}$ & $\mathbf{1 0 0}$ \\
\hline
\end{tabular}

\section{Sex Distribution}

\begin{tabular}{|c|c|c|}
\hline Sex & No. of Patients & Percentage (\%) \\
\hline Male & 17 & 56.66 \\
\hline Female & 13 & 43.37 \\
\hline Total & $\mathbf{3 0}$ & $\mathbf{1 0 0}$ \\
\hline
\end{tabular}

3. Weight

\begin{tabular}{|c|c|c|}
\hline Wt. in kg & No. of Patients & Percentage (\%) \\
\hline $50-59$ & 17 & 56.66 \\
\hline $60-69$ & 08 & 26.67 \\
\hline $70-79$ & 3 & 10.00 \\
\hline $80-90$ & 2 & 6.67 \\
\hline Total & $\mathbf{3 0}$ & $\mathbf{1 0 0}$ \\
\hline
\end{tabular}

4. ASA Grade

\begin{tabular}{|c|c|c|}
\hline ASA Grade & No. of Patients & Percentage (\%) \\
\hline I & 22 & 73.33 \\
\hline II & 8 & 26.67 \\
\hline Total & $\mathbf{3 0}$ & $\mathbf{1 0 0}$ \\
\hline
\end{tabular}

\section{Mallampati Class}

\begin{tabular}{|c|c|c|}
\hline MPC Class & No. of Patients & Percentage (\%) \\
\hline I & 18 & 60 \\
\hline II & 12 & 40 \\
\hline Total & $\mathbf{3 0}$ & $\mathbf{1 0 0}$ \\
\hline
\end{tabular}

\section{Surgeries Performed}

\begin{tabular}{|c|c|}
\hline Surgery & No. of Cases \\
\hline Post Burn Contracture release (hand) & 6 \\
\hline Post Burn Contracture release (leg) & 3 \\
\hline Post Burn scar revision & 3 \\
\hline Claw hand & 3 \\
\hline Scar revision (abdominal wall) & 1 \\
\hline JESS fixator removal (hand) & 1 \\
\hline Split thickness skin grafting (hand) & 6 \\
\hline Split thickness skin grafting (leg) & 3 \\
\hline Split thickness skin grafting (chest) & 2 \\
\hline Debridement & 2 \\
\hline Total & $\mathbf{3 0}$ \\
\hline
\end{tabular}

\section{Ease of Insertion}

\begin{tabular}{|c|c|c|}
\hline Ease of Insertion & No. of Patients & Percentage (\%) \\
\hline Easy & 23 & 76.66 \\
\hline Difficult & 7 & 23.37 \\
\hline Total & $\mathbf{3 0}$ & $\mathbf{1 0 0}$ \\
\hline
\end{tabular}

\section{Insertion Time}

The following graph shows line chart of insertion time in seconds. It ranged from 7 to 48 seconds in our study.

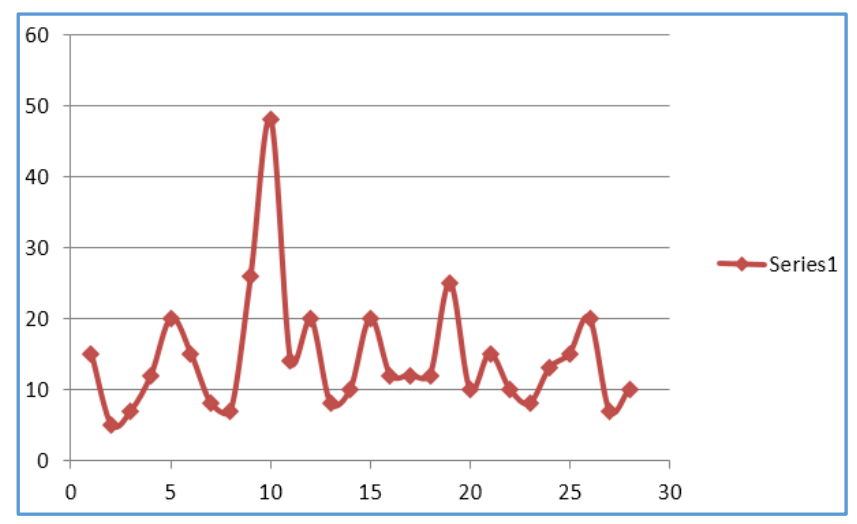

9. Number of Attempts

\begin{tabular}{|c|c|c|}
\hline Number of Attempts & No. of Cases & Percentage (\%) \\
\hline 1 & 21 & 70 \\
\hline 2 & 6 & 20 \\
\hline 3 & 3 & 10 \\
\hline Total & $\mathbf{3 0}$ & $\mathbf{1 0 0}$ \\
\hline
\end{tabular}

There was one case of total failure after 3 attempts.

10. Airway Manoeuvre Requirement

\begin{tabular}{|c|c|c|}
\hline $\begin{array}{c}\text { Airway Manoeuvre } \\
\text { Required }\end{array}$ & No. of Cases & (\%) \\
\hline Yes & 19 & 63.34 \\
\hline No & 11 & 36.66 \\
\hline Total & $\mathbf{3 0}$ & $\mathbf{1 0 0}$ \\
\hline
\end{tabular}

11. Adequacy of Ventilation

\begin{tabular}{|c|c|c|}
\hline Adequacy of Ventilation & No. of Cases & (\%) \\
\hline Adequate & 28 & 93.34 \\
\hline $\begin{array}{c}\text { Not adequate (partial } \\
\text { obstruction) }\end{array}$ & 2 & 6.66 \\
\hline Total & $\mathbf{3 0}$ & $\mathbf{1 0 0}$ \\
\hline
\end{tabular}

12. Gastric Tube Insertion

\begin{tabular}{|c|c|c|}
\hline Gastric Tube Insertion & No. of Cases & $\mathbf{( \% )}$ \\
\hline Easy & 22 & 73.34 \\
\hline Difficult & 05 & 16.66 \\
\hline Failed & 03 & 10 \\
\hline Total & $\mathbf{3 0}$ & $\mathbf{1 0 0}$ \\
\hline
\end{tabular}

13. Adverse Events

\begin{tabular}{|c|c|c|}
\hline Adverse Event & No. of Cases & \% of Cases \\
\hline $\begin{array}{c}\text { Suboptimal oxygenation } \\
\left(\mathrm{SpO}_{2}<95 \%\right)\end{array}$ & 2 & 33.33 \\
\hline Hypoxia $\left(\mathrm{SpO}_{2}<90 \%\right)$ & 0 & 0 \\
\hline Coughing & 1 & 16.67 \\
\hline Laryngospasm & 0 & 0 \\
\hline Leak & 0 & 0 \\
\hline Gastric insufflation & 0 & 0 \\
\hline Hiccups & 0 & 0 \\
\hline Regurgitation & 0 & 0 \\
\hline Aspiration & 0 & 0 \\
\hline Injury to Lip, Teeth, Gum & 0 & 0 \\
\hline Blood on Device & 2 & 33.33 \\
\hline Sore Throat & 1 & 16.67 \\
\hline Dysphonia/Dysphagia & 0 & 0 \\
\hline Nausea/vomiting & 0 & 0 \\
\hline Total & $\mathbf{0 6}$ & $\mathbf{1 0 0}$ \\
\hline
\end{tabular}




\section{RESULTS}

We conducted this study on 30 elective patients posted for plastic surgery. Majority of the patients in our study group 17 $(56.66 \%)$ were in the age group of $20-40$ years while only 2 $(6.67 \%)$ were $<20$ years. 17 patients $(56.66 \%)$ were male, while 13 (43.37\%) were female. All patients were either ASA I or II, with 22 (73.33\%) being ASA grade I and 8 (26.67\%) being ASA grade II. The mouth opening was MPC grade I in 18 (60\%) cases and MPC grade II in 12 (40\%) cases.

The patients were posted for various short duration plastic surgical procedures including Post Burn Contracture release (hand/leg), Post Burn scar revision, Claw hand release, Scar revision, JESS fixator removal, Split thickness skin grafting and debridement.

The insertion of I-gel was easy in 23 (76.66\%) cases while it was difficult in $7(23.37 \%)$ cases. The insertion time ranged from 7 to $48 \mathrm{sec}$. The no. of attempts for insertion were 1 for $21(70 \%)$ cases, 2 for $6(20 \%)$ cases and 3 for $3(10 \%)$ cases. There was one case of total failure after 3 attempts, which we excluded from our study. Airway manoeuvring was required in $19(63.34 \%)$ patients. Ventilation was adequate in 28 (93.34\%) patients, while there was partial obstruction in 2 $(6.66 \%)$ patients. Gastric tube insertion was easy in 22 $(73.34 \%)$ cases, difficult in $05(16.66 \%)$ cases, while it failed in $03(10 \%)$ cases. There were 2 cases $(6.67 \%)$ of suboptimal oxygenation (Sp02<95\%), 1 case $(3.33 \%)$ of coughing, 2 $(6.67 \%)$ cases of blood on device and $1(3.33 \%)$ of sore throat. None of the patients had hypoxia (SpO2<90\%), laryngospasm, leak, gastric insufflation, hiccups, regurgitation, aspiration, injury to lip, teeth, gum, dysphonia, dysphagia, nausea or vomiting.

\section{DISCUSSION}

The study shows that I-gel has many advantages, including ease of insertion, higher success rate on first attempt of insertion, effective airway maintenance, ease of gastric tube insertion, less number of failures and lesser complications intraoperatively as well as postoperatively. All the anaesthetists stated that the placement of I-gel was certainly easier than any other currently available supraglottic device. ${ }^{4}$ This greater stability is primarily related to the anatomical design of the non-inflatable cuff. The ridge at the proximal end of mask catches the base of tongue, thus prevents the device from moving and so contributes to the positional stability of the device after placement. ${ }^{5}$ Since no cuff inflation is needed in this device, there is shorter time to achieve effective airway.

I-gel has been found to have easiest insertion in various types of manikins. ${ }^{6}$ R. M. Levitan and W. C. Kinkle in 2005, studied the positioning and mechanics of I-gel in 65 nonembalmed cadavers with 73 endoscopies. They found that the I-gel effectively conformed to the perilaryngeal anatomy despite the lack of an inflatable cuff and it consistently achieved proper positioning for supraglottic ventilation.

Mirko Gutzmann, M.D., Jacqueline Roemer ${ }^{7}$ et al conducted a Prospective Multicentre Clinical Evaluation of the cuffless supraglottic airway device I-gel ${ }^{\text {TM }}$ from March 2008. Out of the 974 patients evaluated, insertion success rate was 95\%, simplicity of placement was easy in $72 \%$ and impossible in $0.2 \%$, gastric catheter placement was successful in $90 \%$, blood stain on device was found in $4 \%$ and laryngeal/bronchogenic spasm occurred in $0.3 \%$. They concluded that the I-gel is a highly successful and easy-to-place supraglottic airway device providing sufficient airway seal to allow controlled ventilation in a large number of patients in a large variety of settings at a very low incidence of complication.

V. Uppal, G. Fletcher and J. Kinsella ${ }^{8}$ conducted a study to compare I-gel with the cuffed tracheal tube during pressurecontrolled ventilation. They concluded that I-gel can be used as a reasonable alternative to tracheal tube during PCV with moderate airway pressures.

\section{CONCLUSION}

I-gel is a simple, excellent and easy-to-use supraglottic airway device, easy to insert without the need of many manipulations with maintenance of airway. It is easy to insert without need of many manipulations with maintenance of airway in a short time. The device is very effective and useful for adult patients requiring surgical procedures of 30-60 minutes duration while breathing spontaneously.

\section{REFERENCES}

[1] Brain AI, McGhee TD, McAteer EJ, et al. The laryngeal mask airway. Development and preliminary trials of a new type of airway. Anaesthesia 1985;40(4):356-61.

[2] Bein B, Scholz J. Supraglottic airway devices. Best Pract Res Clin Anaesthesiol 2005;19(4):581-93.

[3] Rabey PG, Murphy PJ, Langton JA, et al. Effect of the laryngeal mask airway on lower oesophageal sphincter pressure in patients during general anaesthesia. $\mathrm{Br} \mathrm{J}$ Anaesth 1992;69:346-8.

[4] Kannaujia A, Srivastava U, Saraswat N, et al. A preliminary study of I-gel: a new supraglottic airway device. Indian Journal of Anaesthesia 2009;53(1):52-6.

[5] Levitan RM, Kinkle WC. Initial anatomic investigation of the I-gel airway: a novel supraglottic airway without inflatable cuff. Anaesthesia 2005;60(10):1022-6.

[6] Jackson KM, Cook TM. Evaluation of four airway training manikins as patient simulators for the insertion of eight types of supraglottic airway devices. Anaesthesia 2007;62(4):388-93.

[7] Gutzmann M, Roemer J, Theiler L, et al. Prospective multicenter clinical evaluation of the cuffless supraglottic airway device I-gel ${ }^{\mathrm{TM}}$. American Society of Anaesthesiologists 2009.

[8] Uppal V, Fletcher G, Kinsella J. Comparison of the I-gel with the cuffed tracheal tube during pressurecontrolled ventilation. British Journal of Anaesthesia 2009;102(2):264-8. 